\title{
Effects of Bridge Functions on Radial Distribution Functions of Liquid Water
}

\author{
Shigenori TANAKA* \\ Graduate School of System Informatics, Kobe University, \\ 1-1 Rokkodai, Nada-ku, Kobe 657-8501, Japan \\ Miki NAKANO \\ Frontier Institute for Biomolecular Engineering Research (FIBER), Konan University, \\ 7-1-20 Minatojima-minamimachi, Chuo-ku, Kobe 650-0047, Japan
}

(Dated: January 25, 2020)

\begin{abstract}
In this report the radial distribution functions (RDFs) of liquid water are calculated on the basis of the classical density functional theory combined with the reference interaction site model for molecular liquids. The bridge functions, which are neglected in the hypernetted-chain (HNC) approximation, are taken into account through the density expansion for the Helmholtz free energy functional up to the third order. A factorization approximation to the ternary direct correlation functions in terms of the site-site pair correlation functions is then employed in the expression of the bridge functions, thus leading to a closed set of integral equations for the determination of the RDFs. It is confirmed through numerical calculations that incorporation of the oxygen-oxygen bridge function substantially improves the poor descriptions by the HNC approximation at room temperature, e.g., for the second peak of the oxygen-oxygen RDF.
\end{abstract}

Key words: water, radial distribution function, density functional theory, reference interaction site model, bridge function

*Electronic address: tanaka2@kobe-u.ac.jp 


\section{INTRODUCTION}

Integral equation approach provides an efficient tool to calculate the correlation and thermodynamic functions of liquids with high accuracy and mild cost of computation (Hansen and McDonald, 2006; Ichimaru et al., 1987). This analytical approach often gives fair results on par with those by extensive Monte Carlo or molecular dynamics (MD) simulations, and combined with the reference interaction site model (RISM) method (Chandler and Andersen, 1972; Hansen and McDonald, 2006; Hirata and Rossky, 1981), can comprehensively describe the equilibrium properties of molecular liquids such as liquid water (Pettitt and Rossky, 1982), which plays essential roles in a variety of biochemical processes. Recent developments in the methods, algorithms and benchmarks (Lombardero et al., 1999; Lue and Blankschtein, 1995; Reddy et al., 2003; Richardi et al., 1999; Sato, 2013; Sumi and Sekino, 2006) indicate that the RISM-based integral equation approach can provide an alternative route for theoretical analyses on water and related aqueous systems with comparable reliability to more expensive, computer simulation approaches. However, it has also been observed (Lombardero et al., 1999; Lue and Blankschtein, 1995; Reddy et al., 2003; Richardi et al., 1999; Sato, 2013; Sumi and Sekino, 2006) that the descriptions of intermolecular correlations of water become less accurate at room temperature in comparison with at higher temperatures.

Among the RISM-based integral equation formalisms for molecular liquids, the density functional theory (DFT) approach due to Chandler, McCoy and Singer (Chandler et al., 1986) is one of the most sophisticated and dependable methods. Donley, Curro and McCoy (DCM) (Donley et al., 1994) then extended this DFT scheme for the calculations of pair correlation functions of molecular liquids, and later, Reddy et al. (Reddy et al., 2003) and Sumi et al. (Sumi and Sekino, 2006) applied this method to the calculations of the radial distribution functions (RDFs) of fluid water. Their calculated results demonstrated that the DCM scheme provides accurate descriptions at high temperatures, but the deviations from the computer simulation results are observed at room temperature, e.g., in the description 
of the location of the second peak of oxygen-oxygen (O-O) RDF, which may be associated with the formation of tetrahedral structure of condensed water. Considering that the DCM theory is based on the density expansion of free energy functional up to the second order, which corresponds to the hypernetted-chain (HNC) approximation (Hansen and McDonald, 2006; Ichimaru et al., 1987) in liquid theory, the present authors (Tanaka and Nakano, 2013) have recently extended the DCM theory so as to retain the density expansion of free energy functional up to the third order, thus taking into account the effect of the bridge functions (Hansen and McDonald, 2006; Ichimaru et al., 1987) beyond the HNC approximation. Their preliminary test calculation in which a simple Gaussian approximation is employed in the factorization (Barrat et al., 1988) for the ternary direct correlation functions in the bridge functions has, however, failed to appropriately reproduce the simulation results for liquid water at room temperature, suggesting the importance of incorporation of detailed structures of correlation functions in the representation of ternary direct correlation functions. The present work then investigates another factorization approximation for the bridge functions, in which the site-site pair correlation functions are employed in the factorization according to a scheme (Iyetomi and Ichimaru, 1983) that worked well in the one-component plasma system (Ichimaru et al., 1987).

\section{METHODS}

Let us consider a liquid water system consisting of rigid $\mathrm{H}_{2} \mathrm{O}$ molecules whose average number density and temperature are $\rho$ and $T$, respectively. We then assume the site-site interaction potentials $v_{\alpha \beta}(\boldsymbol{r})$ between the atomic sites $\alpha$ and $\beta(\alpha, \beta=\mathrm{O}, \mathrm{H}, \mathrm{H}$, i.e., one oxygen and two hydrogens) on two water molecules. According to the DFT scheme (Donley et al., 1994; Tanaka and Nakano, 2013), the RDF between the sites $\alpha$ and $\beta$ is given by

$$
g_{\alpha \beta}\left(\boldsymbol{r}_{\alpha}-\boldsymbol{R}_{\beta}\right)=\left\langle\left\langle\exp \left[-\beta \sum_{\eta, \gamma} v_{\eta \gamma}\left(\boldsymbol{r}_{\eta}-\boldsymbol{R}_{\gamma}\right)+\Lambda(\{\boldsymbol{r}\},\{\boldsymbol{R}\})+B(\{\boldsymbol{r}\},\{\boldsymbol{R}\})\right]\right\rangle\right\rangle_{\boldsymbol{r}_{\alpha}, \boldsymbol{R}_{\beta}}^{\mathcal{P}}
$$


with $\beta=1 / k_{\mathrm{B}} T$ and $k_{\mathrm{B}}$ being the Boltzmann constant, where $\langle\langle\rangle\rangle_{\boldsymbol{r}_{\alpha}, \boldsymbol{R}_{\beta}}^{\mathcal{P}}$ refers to the avarage over the relative orientation of two water molecules whose $\alpha$ th and $\beta$ th sites are fixed at $\boldsymbol{r}_{\alpha}$ and $\boldsymbol{R}_{\beta}$, respectively, and whose coordinates are represented by $\{\boldsymbol{r}\}=\left\{\boldsymbol{r}_{\eta}\right\}$ and $\{\boldsymbol{R}\}=\left\{\boldsymbol{R}_{\gamma}\right\}$, respectively. In this expression, the two-body part is given by

$$
\Lambda(\{\boldsymbol{r}\},\{\boldsymbol{R}\})=\rho \sum_{\eta, \gamma} \sum_{\sigma, \xi} \int d \boldsymbol{r}_{\sigma} \int d \boldsymbol{r}_{\xi} c_{\eta \sigma}\left(\boldsymbol{r}_{\eta}-\boldsymbol{r}_{\sigma}\right) h_{\sigma \xi}\left(\boldsymbol{r}_{\sigma}-\boldsymbol{r}_{\xi}\right) \omega_{\xi \gamma}^{-1}\left(\boldsymbol{r}_{\xi}-\boldsymbol{R}_{\gamma}\right)
$$

with the aid of a site-site pairwise approximation for the induced density (Donley et al., 1994), where

$$
\omega_{\alpha \beta}(\boldsymbol{r})=\delta_{\alpha \beta} \delta(\boldsymbol{r})+\left(1-\delta_{\alpha \beta}\right) \delta\left(r-L_{\alpha \beta}\right) / 4 \pi L_{\alpha \beta}^{2}
$$

with $L_{\alpha \beta}$ being the distance between sites $\alpha$ and $\beta$ means the intramolecular correlation functions. The site-site pair correlation functions $h_{\alpha \beta}(r)=g_{\alpha \beta}(r)-1$ and the two-body direct correlation functions $c_{\alpha \beta}(r)$ are then related via the site-site Ornstein-Zernike or RISM equation (Chandler and Andersen, 1972; Hansen and McDonald, 2006),

$$
h_{\alpha \beta}(r)=\sum_{\eta, \sigma}\left[\omega_{\alpha \eta} * c_{\eta \sigma} * \omega_{\sigma \beta}(r)+\rho \omega_{\alpha \eta} * c_{\eta \sigma} * h_{\sigma \beta}(r)\right],
$$

where $*$ means the convolution. If we neglect the higher-order contribution $B(\{\boldsymbol{r}\},\{\boldsymbol{R}\})$ in Eq. (1), we are led to a two-body approximation that is equivalent to the HNC approximation (Hansen and McDonald, 2006; Ichimaru et al., 1987). The HNC solutions to the integral equations for the correlation functions of fluid water were obtained and compared to the computer simulation and experimental results in the literature (Reddy et al., 2003; Sumi and Sekino, 2006; Tanaka and Nakano, 2013). In contrast to successful descriptions at higher temperatures, one then observed a significant discrepancy between the HNC and MD results at room temperature, in particular concerning the location of the second peak of $g_{O O}(r)$ that was situated at $r \simeq 5.5 \AA$ in the HNC result in comparison with $r \simeq 4.5 \AA$ in the MD and experimental results. This observation indicates that the O-O tetrahedral ordering in dense, 
low-temperature water systems is not appropriately described by the second-order, HNC approximation for the intermolecular correlations.

Then, we proceed to an incorporation of the triplet correlation term in Eq. (1). In a preceding DFT study (Tanaka and Nakano, 2013) we derived an explicit expression for $B(\{\boldsymbol{r}\},\{\boldsymbol{R}\})$ in terms of ternary direct correlation functions, and employed, as a first attempt, a simple Gaussian approximation for the factorized, three-body direct correlation functions. However, the calculated result for $g_{\alpha \beta}(r)$ failed to quantitatively reproduce the MD or experimental results. In the present study we instead consider another factorization approximation according to Iyetomi and Ichimaru (Iyetomi and Ichimaru, 1983), which took relevant account of the lowest-order contribution and worked well in the case of strongly coupled, one-component plasma (Ichimaru et al., 1987). It is expected that this approximation would provide a good description for hard and soft sphere systems as well as for Coulombic system, considering the universality in strongly correlated systems (Hansen and McDonald, 2006; Ichimaru et al., 1987). Taking into account only the O-O contribution, which would play a primary role, the bridge function can then be given by

$$
B_{O O}(r)=\frac{1}{2} \rho^{2} \int d \boldsymbol{r}^{\prime} \int d \boldsymbol{r}^{\prime \prime} h_{O O}\left(\boldsymbol{r}-\boldsymbol{r}^{\prime}\right) h_{O O}\left(\boldsymbol{r}^{\prime}-\boldsymbol{r}^{\prime \prime}\right) h_{O O}\left(\boldsymbol{r}^{\prime \prime}-\boldsymbol{r}\right) h_{O O}\left(r^{\prime}\right) h_{O O}\left(r^{\prime \prime}\right)
$$

with $r=|\boldsymbol{r}|=\left|\boldsymbol{r}_{O}-\boldsymbol{R}_{O}\right|$ for $B(\{\boldsymbol{r}\},\{\boldsymbol{R}\})$ in Eq. (1), which is symmetric with respect to $\boldsymbol{r}_{O}$ and $\boldsymbol{R}_{O}$ due to the exclusive consideration of O-O correlation.

However, there is a drawback in the expression of Eq. (5) that this form of bridge function does not reproduce the correct short-range bahavior due to $h_{\alpha \beta}(r) \rightarrow-1$ at $r \rightarrow 0$ (Barrat et al., 1988; Iyetomi and Ichimaru, 1983). We hence renormalize the bridge function as (Tanaka and Nakano, 2013)

$$
\tilde{B}(r)=f(r) B_{O O}(r)
$$

by introducing a short-range enhancement function (Iyetomi and Ichimaru, 1983) as 


$$
f(r)=(C-1) \exp \left[-\left(\frac{r}{a}\right)^{2}\right]+1 .
$$

The parameters in Eq. (7) are roughly fixed as $a=4.5 \AA$ and $C=30.0$ in the following analysis, considering the location of the second peak of $g_{O O}(r)$ for the former and the typical magnitude of the short-range renormalization for $h_{\alpha \beta}(r)$ (Barrat et al., 1988; Iyetomi and Ichimaru, 1983) for the latter. In addition, to reduce the computational cost, we use the experimental results (Soper, 2000) for $h_{O O}(r)$ to evaluate $B_{O O}(r)$ in terms of Eq. (5). The behavior of $\tilde{B}(r)$ at $\rho=0.0334 \AA^{-3}$ and $T=300 \mathrm{~K}$ is depicted in Fig. 1 .

\section{RESULTS AND DISCUSSION}

We have employed the SPC/E model (Berendsen et al., 1987) for the (rigid) structure and intermolecular potentials for water molecules, thus assuming a three-site (one oxygen and two hydrogens) model in which the Lennard-Jones potential works only between the oxygen $(\mathrm{O})$ sites. We have thus carried out the calculation of the RDFs of liquid water at $\rho=0.0334 \AA^{-3}$ and $T=300 \mathrm{~K}$, where the renormalized bridge function, $\tilde{B}(r)$, above was employed in Eq. (1). The computational details are found in the literature (Tanaka and Nakano, 2013). The calculated results for $g_{\alpha \beta}(r)$ are shown in Fig. 2, in which we have also depicted the results by $10 \mathrm{~ns}$ MD simulation (Tanaka and Nakano, 2013) for the SPC/E water performed with $A M B E R$ 12 software (Case et al., 2012) as well as those by a neutron diffraction experiment (Soper, 2000) for comparison.

It is seen in Fig. 2 (b) and (c) that the present DFT scheme with inclusion of $\tilde{B}(r)$ reproduces the behaviors of $g_{O H}(r)$ and $g_{H H}(r)$ obtained in the MD simulation fairly well. Concerning the description of $g_{O O}(r)$ seen in Fig. $2(\mathrm{a})$, on the other hand, the present DFT scheme slightly improves the behavior of the second peak of $g_{O O}(r)$ over the HNC result (Reddy et al., 2003; Sumi and Sekino, 2006; Tanaka and Nakano, 2013), yielding the (weak) peak at $r \simeq 4.7 \AA$ in comparison with that at $r \simeq 4.5 \AA$ in the MD result and $r \simeq 5.5 \AA$ in the HNC result. It should be remarked, however, that the agreement between the DFT 
and MD results is still unsatisfactory especially concerning the quantitative description of the oscillatory behavior of $g_{O O}(r)$, e.g., the location of the third peak, thus needing further improvement on the theoretical scheme beyond the factorization approximation in terms of $h_{O O}(r)$. For instance, the contributions other than the O-O correlation may play some roles in $B(\{\boldsymbol{r}\},\{\boldsymbol{R}\})$. In addition, there is a possibility that the fourth- and higher-order terms contribute to the bridge functions to some extent.

In this context, we have attempted to correct $\tilde{B}(r)$ in an $a d$ hoc way by

$$
\delta \tilde{B}(r)=D\left\{\exp \left[-\left(\frac{r-r_{1}}{\Delta_{1}}\right)^{2}\right]-\exp \left[-\left(\frac{r-r_{2}}{\Delta_{2}}\right)^{2}\right]-\exp \left[-\left(\frac{r-r_{3}}{\Delta_{3}}\right)^{2}\right]\right\}
$$

with $D=0.1, r_{1}=4.5 \AA, r_{2}=3.5 \AA, r_{3}=5.5 \AA$, and $\Delta_{1}=\Delta_{2}=\Delta_{3}=0.5 \AA$, considering the discrepancy between the DFT and MD results for $g_{O O}(r)$ observed in Fig. 2. We have thus performed the calculation of RDFs with the corrected bridge function,

$$
\tilde{B}^{c}(r)=\tilde{B}(r)+\delta \tilde{B}(r)
$$

While the correction $\delta \tilde{B}(r)$ is very minor as seen in Fig. 1 (a) (indiscernible between $\tilde{B}(r$ ) and $\tilde{B}^{c}(r)$ at the full scale), we have found that this correction substantially improves the agreement between the DFT and MD results as observed in Fig. 2, which indicates a delicate manner (see Fig. 1 (b) at the zoomed scale) to quantitatively describe the RDFs with the bridge functions at medium to long distances. More accurate descriptions of the RDFs for liquid water would thus be a challenging task in the framework of many-body theory.

\section{CONCLUSION}

In summary, we calculated the RDFs of liquid water at ambient temperature and density on the basis of the third-order DFT method, in which the ternary direct correlation functions were expressed by the factorization with the pair correlation functions and the O-O bridge function was taken into account beyond the $\mathrm{HNC}$ approximation in the expression for the 
RDFs. Improvement on the HNC scheme was thus achieved with the use of the proposed bridge function and its corrected one, especially concerning the behavior of $g_{O O}(r)$, while some future tasks still remain for better theoretical description.

\section{Acknowledgment}

S.T. would like to acknowledge the Grants-in-Aid for Scientific Research (No. 23540451)

from the Ministry of Education, Cultute, Sports, Science and Technology (MEXT). 
[1] Barrat, J.L., Hansen, J.P., Pastore, G. 1988. On the equilibrium structure of dennse fluids: Triplet correlations, integral equations and freezing. Mol Phys 63, 747-767.

[2] Berendsen, H.J.C., Grigera, J.R., Straatsma, T.P. 1987. The missing term in effective pair potentials. J Phys Chem 91, 6269-6271.

[3] Case, D.A. et al. 2012. AMBER 12. University of California, San Francisco.

[4] Chandler, D., Andersen, H.C. 1972. Optimized cluster expansions for classical fluids. II. Theory of molecular liquids. J Chem Phys 57, 1930-1937.

[5] Chandler, D., McCoy, J.D., Singer, S.J. 1986. Density functional theory of nonuniform polyatomic systems. I. General formulation. J Chem Phys 85, 5971-5976.

[6] Chandler, D., McCoy, J.D., Singer, S.J. 1986. Density functional theory of nonuniform polyatomic systems. II. Rational closures for integral equations. J Chem Phys 85, 5977-5982.

[7] Donley, J.P., Curro, J.G., McCoy, J.D. 1994. A density functional theory for pair correlation functions in molecular liquids. J Chem Phys 101, 3205-3215.

[8] Hansen, J.-P., McDonald, I.R. 2006. Theory of Simple Liquids., 3rd ed. Academic Press, London.

[9] Hirata, F., Rossky, P.J. 1981. An extended RISM equation for molecular polar fluids. Chem Phys Lett 83, 329-334.

[10] Ichimaru, S., Iyetomi, H., Tanaka, S. 1987. Statistical physics of dense plasmas: Thermodynamics, transport coefficients and dynamic correlations. Phys Rep 149, 91-205.

[11] Iyetomi, H., Ichimaru, S. 1983. Density-functional theory of correlations in dense plasmas: Improvement on the hypernetted-chain scheme. Phys Rev A 27, 3241-3250.

[12] Lombardero, M., Martin, C., Jorge, S., Lado, F., Lomba, E. 1999. An integral equation study of a simple point charge model of water. J Chem Phys 110, 1148-1153.

[13] Lue, L., Blankschtein, D. 1995. Application of integral equation theories to predict the structure, thermodynamics, and phase behavior of water. J Chem Phys 102, 5427-5437.

[14] Pettitt, B.M., Rossky, P.J. 1982. Integral equation predictions of liquid state structure for waterlike intermolecular potentials. J Chem Phys 77, 1451-1457.

[15] Reddy, G., Lawrence, C.P., Skinner, J.L., Yethiraj, A. 2003. Liquid state theories for the structure of water. J Chem Phys 119, 13012-13016. 
[16] Richardi, J., Millot, C., Fries, P.H. 1999. A molecular Ornstein-Zernike study of popular models for water and methanol. J Chem Phys 110, 1138-1147.

[17] Sato, H. 2013. A modern solvation theory: Quantum chemistry and statistical chemistry. Phys Chem Chem Phys 15, 7450-7465.

[18] Soper, A.K. 2000. The radial distribution functions of water and ice from 220 to $673 \mathrm{~K}$ and at pressures up to $400 \mathrm{MPa}$. Chem Phys 258, 121-137.

[19] Sumi, T., Sekino, H. 2006. An interaction site model integral equation study of molecular fluids explicitly considering the molecular orientation. J Chem Phys 125, 034509.

[20] Tanaka, S., Nakano, M. 2013. Triplet correlations and bridge functions in classical density functional theory for liquid water. Chem Phys Lett 572, 38-43. 


\section{Figure caption}

Fig. 1. Oxygen-oxygen bridge functions $\tilde{B}(r)$ (red dashed line) and $\tilde{B}^{c}(r)$ (blue solid line) for liquid water at $\rho=0.0334 \AA^{-3}$ and $T=300$ K. (a) Full scale. (b) Zoomed scale.

Fig. 2. Radial distribution functions (RDFs) obtained by the DFT scheme with inclusion of bridge function $\tilde{B}(r)$ (red dotted line), that with $\tilde{B}^{c}(r)$ (blue solid line), the MD simulation (Tanaka and Nakano, 2013) (green dashed lines), and the neutron diffraction experiment (Soper, 2000) (circles) for liquid water at $\rho=0.0334 \AA^{-3}$ and $T=300 \mathrm{~K}$. (a) $g_{O O}(r)$; (b) $g_{O H}(r) ;(\mathrm{c}) g_{H H}(r)$. 